\title{
Selective detection of dopamine in the presence of ascorbic acid via fluorescence quenching of $\mathrm{InP} / \mathrm{ZnS}$ quantum dots
}

This article was published in the following Dove Press journal:

International Journal of Nanomedicine

25 August 2015

Number of times this article has been viewed

\section{Seshadri Reddy Ankireddy Jongsung Kim}

Department of Chemical and Biological Engineering, Gachon University, Seongnam, South Korea
Correspondence: Jongsung Kim Department of Chemical and Biological Engineering, Gachon University, Seongnam, Gyeonggi-Do, 46I-70I, South Korea

Tel +82 I0 22|2 536।

Fax +82317505363

Email jongkim@gachon.ac.kr

\begin{abstract}
Dopamine is a neurotransmitter of the catecholamine family and has many important roles, especially in human brain. Several diseases of the nervous system, such as Parkinson's disease, attention deficit hyperactivity disorder, restless legs syndrome, are believed to be related to deficiency of dopamine. Several studies have been performed to detect dopamine by using electrochemical analysis. In this study, quantum dots (QDs) were used as sensing media for the detection of dopamine. The surface of the QDs was modified with L-cysteine by coupling reaction to increase the selectivity of dopamine. The fluorescence of cysteine-capped indium phosphide/zinc sulfide QDs was quenched by dopamine with various concentrations in the presence of ascorbic acid. This method shows good selectivity for dopamine detection, and the detection limit was $5 \mathrm{nM}$
\end{abstract}

Keywords: InP/ZnS QDs, Parkinson's disease, L-dopamine, ascorbic acid, neurotransmitter, photoluminescence

\section{Introduction}

During the past two decades, a lot of attention has been paid to quantum dots (QDs) nanocrystalline semiconductors because of their potential applications in optoelectronics. Significant progress has been achieved in the preparation and application of QDs. Several kinds of QDs of various shapes and structures have been prepared, and a lot of work has been done for the characterization of QDs. When QDs are excited by photons, electron-hole pairs are formed, and their recombination leads to strong fluorescence. QDs have several unusual optical properties, such as size-dependent emission, color with narrow emission band, high fluorescence intensity, and high stability against photo bleaching that enables many applications in light-emitting diodes, ${ }^{1,2}$ sensors, ${ }^{3}$ and biomedical labels. ${ }^{4,5}$

Dopamine (3,4-dihydroxyphenethylamine) (DA) is a neurotransmitter of the catecholamine family, which is widely distributed in the central nervous system of animals for message transfer from one neuron to another neuron. ${ }^{6,7}$ It is derived from L-tyrosine by a series of biochemical reactions using tyrosine hydroxylase and decarboxylase, and it is the precursor to norepinephrine (noradrenaline) and epinephrine (adrenalin). DA is also a hormone that is related to many important functions in the human brain and body. ${ }^{8-10}$ It is closely related to brain functions, such as motivation, sleep, attention, and learning. The malfunction of the dopamine system results in several brain diseases, such as attention deficit hyperactivity disorder, restless legs syndrome, schizophrenia, Parkinson's, and Alzheimer's disease. ${ }^{11-14}$ The DA concentration is quite low in the nerve cell. In the extracellular fluid of the central nervous system and cerebrospinal 
fluid, DA concentration is $<100 \mathrm{nM}^{15}$ and also, depending on location, the concentration of DA ranges broadly from $1.0 \times 10^{-7} \mathrm{M}$ to $1.0 \times 10^{-3} \mathrm{M} .^{16}$

Several methods have been implemented to detect DA, such as electrogenerated chemiluminescence, colorimetry, capillary electrophoresis, fluorescence spectrometry, and high-performance liquid chromatography. ${ }^{17-23}$ A lot of attention has been paid to fluorescence spectrometry of QDs due to its simplicity, easiness, and high accuracy in the detection of DA, especially at low concentration levels. However, the detection of DA by fluorescence in biological matrices usually suffers from interference by ascorbic acid (AA). Thus, it is important to design a new method for the determination of DA in the presence of AA with high selectivity and sensitivity.

A few studies have been performed on the detection of DA using QDs. Li et al detected dopamine based on its quenching effect on the anodic electrochemiluminescence of CdSe QDs. ${ }^{24}$ Zhao et al have used (3-aminopropyl) triethoxysilane (APTES)-capped ZnO QDs for the direct detection of dopamine. ${ }^{25}$ However, CdSe QDs would eventually release heavy metal $\mathrm{Cd}$ ions, and APTES is a toxic compound that is destructive to the mucous membranes and targets organs such as the nerves, liver, and kidneys.

In this work, we have prepared water-soluble L-cysteine (L-Cys)-capped indium sulphide/zinc sulfide ( $\mathrm{InP} / \mathrm{ZnS}$ ) QDs for the detection of DA. InP/ZnS QDs do not have any heavy metal ions and thus are eco-friendly. L-Cys, one of the natural amino acids, is also more biocompatible than APTES. L-Cys-capped InP/ZnS QDs were prepared by coupling reaction to produce disulfide bond, which converted the InP/ZnS QDs from hydrophobic to hydrophilic. The effect of AA and DA on the fluorescence quenching of the QDs was compared. The fluorescence quenching of the QDs with various concentrations of DA in the presence of AA was investigated.

\section{Materials and Methods Chemicals and instruments}

$\mathrm{InP} / \mathrm{ZnS}$ QDs in toluene $(5 \mathrm{mg} / \mathrm{mL}$, emission wavelength at $580 \mathrm{~nm}$ ) were purchased from Mesolight (People's Republic of China). L-Dopamine hydrochloride, AA, L-Cys, and toluene were purchased from Sigma Aldrich, and ethanol (EtOH) was purchased from Duksan Pure Chemicals. All reagents were used without any further purification.

Photoluminescence (PL) spectra of QDs were obtained by using fluoroluminescence spectrometer (Quanta Master, Photon Technology International, NJ, USA) equipped with a xenon lamp (Arc Lamp Housing, A-1010B ${ }^{\mathrm{TM}}$ ), monochromator, and power supply (Brytexbox). Absorption spectra of QDs were obtained using a Cary 100 UV/Vis spectrophotometer (Varian). The FTIR spectra were recorded using a Bruker vortex 70 FTIR spectrometer. Sonication processes were done with Branson 8510 sonicator.

\section{Surface modification of $\operatorname{lnP} / \mathrm{ZnS}$ QDs with L-cysteine}

The surface modification of InP/ZnS QDs with L-Cys was done by using previously reported work by Ariffin et al. ${ }^{26}$ A total of $1 \mathrm{~mL}$ of $\mathrm{InP} / \mathrm{ZnS}$ QDs was dissolved in $1 \mathrm{~mL}$ of toluene and ethanol, purified by centrifugation at 3,000 rpm for 15 minutes, and washed with ethanol. The purification process was repeated three times, and the precipitate was collected and redissolved in toluene. An excess amount of thiol-terminated L-Cys was added into the QDs solution, and the mixture was sonicated for 30 minutes. After sonication, the reaction mixture was kept overnight at room temperature. Then the mixture was centrifuged at 5,000 rpm for 20 minutes and washed with ethanol. The supernatant was removed and kept dried under vacuum in desiccator for 1 hour to yield water-soluble L-Cys-capped InP/ZnS QDs.

\section{Detection of dopamine}

Dopamine can be detected in the presence of AA by fluorescence quenching of cysteine-capped InP/ZnS QDs. A total of $700 \mu \mathrm{L}$ of diluted solution of cysteine-capped InP/ZnS QDs was mixed with $500 \mu \mathrm{M}$ of AA and various concentrations of DA ( $5 \mathrm{nM}, 10 \mathrm{nM}, 20 \mathrm{nM}, 40 \mathrm{nM}, 80 \mathrm{nM}, 100 \mathrm{nM}$ in deionized water). The solution was mixed thoroughly, and after incubation for 5 minutes, PL spectra were measured at an excitation wavelength of $349 \mathrm{~nm}$.

\section{Results and discussion}

The InP/ZnS QDs were insoluble in the DI water and appeared in the organic phase. After surface modification with L-Cys amino acid, these QDs were readily soluble in DI water and appeared in the water phase. Water-soluble L-Cys-capped InP/ZnS QDs were obtained. High-resolution transmission electron microscopy (HRTEM) was employed to characterize the size and morphology of water-soluble L-Cys-capped InP/ZnS QDs. The QDs were mixed with $1 \mathrm{~mL}$ ethanol and sonicated for 10 minutes. After sonication, a few drops of InP/ZnS QDs were placed on to carbon-coated copper grids, allowing the excess solvent to evaporate for HRTEM analysis. Figure 1 shows the HRTEM image of L-Cys-capped InP/ZnS QDs. The figure shows that the QDs are monodispersed with an average diameter of 3-5 nm. The inset shows a clear enlarged image of the single InP/ $\mathrm{ZnS}$ nanocrystals. 


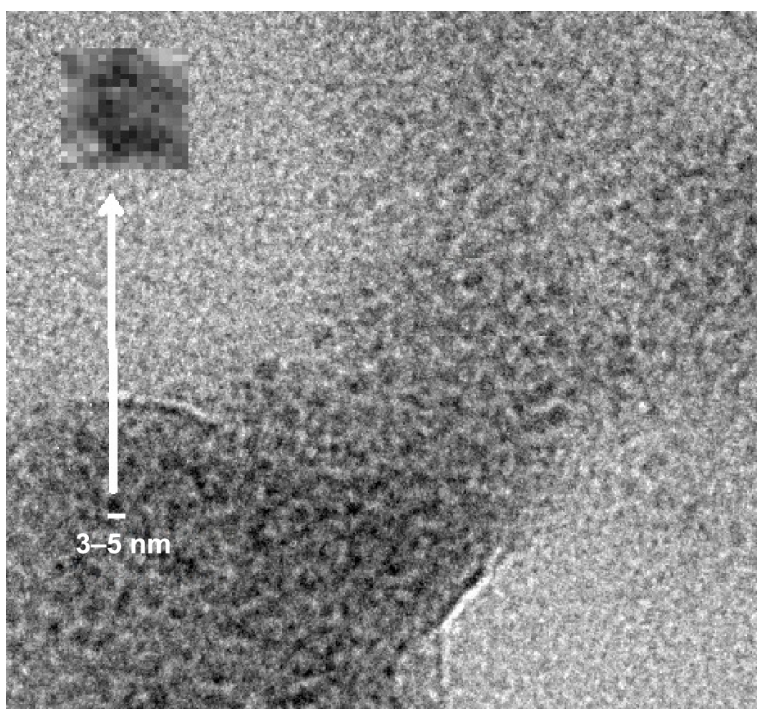

Figure I The HRTEM image of the L-Cys-capped InP/ZnS QDs.

Note: The inset shows a clear enlarged image of a single InP/ZnS nanocrystal. Abbreviations: HRTEM, high-resolution transmission electron microscopy; L-Cys, L-cysteine; InP/ZnS QDs, indium phosphide/zinc sulfide quantum dots.

The FTIR measurements were performed on the surfacemodified QDs powder in order to confirm the binding of the capping molecules on the surface of the InP/ZnS QDs. Figure 2 shows the FTIR spectra of (A) free L-Cys and (B) L-Cys-capped InP/ZnS QDs. In the figure, the intense band at 3,245 $\mathrm{cm}^{-1}$ was assigned to the amine $\left(-\mathrm{NH}_{2}\right)$ functional group of L-Cys. The absorption peak at 2,957 $\mathrm{cm}^{-1}$ was assigned to $v_{\text {aliphatic }}(\mathrm{CH})$ of the L-Cys. Characteristic peaks at $1,582 \mathrm{~cm}^{-1}, 1,460 \mathrm{~cm}^{-1}, 1,420 \mathrm{~cm}^{-1}$, and $1,098 \mathrm{~cm}^{-1}$ were assigned to $v_{\text {asym }}(\mathrm{COO}), \delta(\mathrm{NH})$ bending, $v_{\text {sym }}(\mathrm{COO})$, and $v(\mathrm{C}-\mathrm{N})$ of L-Cys. The peak at $2,550 \mathrm{~cm}^{-1}$ represents the $\delta(\mathrm{S}-\mathrm{H})$ stretching vibration of L-Cys. However, this

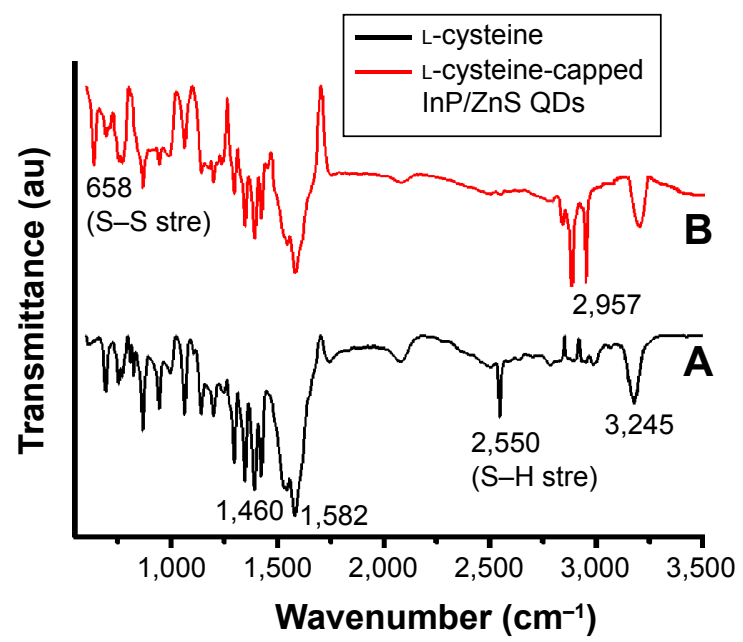

Figure 2 The FTIR spectra of (A) free L-cysteine and (B) L-cysteine-capped InP/ ZnS QDs.

Abbreviations: stre, stretching; FTIR, Fourier transform infrared spectroscopy; InP/ZnS QDs, indium phosphide/zinc sulfide quantum dots; DI, deionized. characteristic peak of S-H was not observed in Figure 2B, which indicates that the $\mathrm{S}-\mathrm{H}$ bond was cleaved and that a new S-S bond was formed between the L-Cys and InP/ZnS QDs. In Figure 2B, the peak at $658 \mathrm{~cm}^{-1}$ was attributed to $\delta(\mathrm{S}-\mathrm{S})$ stretching vibrations; however, this peak was not observed in L-Cys.

The optical absorption and normalized PL spectra of InP/ $\mathrm{ZnS}$ QDs before and after surface modification with L-Cys are shown in Figure 3A and B. InP/ZnS QDs were dispersed in toluene, while L-Cys-modified QDs were dispersed in DI water. Inset is the photograph of the QDs, before and after surface modification, dispersed in two-layered mixture of toluene and DI water under UV illumination. The figure shows that absorption and PL peaks of the QDs were redshifted by the surface modification, which may be due to the interaction between solute and solvent molecules to form $\mathrm{n}-\pi^{*}$ transitions apart from $\pi-\pi *$ transitions. In the excited state, the solvent dipoles can reorient or relax around, which lowers the energy of the excited electrons. As the solvent polarity is increased, this effect becomes larger, resulting in redshift. The variation of PL spectra of cysteine-capped $\mathrm{InP} / \mathrm{ZnS}$ QDs by the addition of $500 \mu \mathrm{M}$ of AA (A) and plot of normalized fluorescence intensity versus time (B) are shown in Figure 4. When AA solution was added into the reaction mixture, the AA molecules were absorbed on QDs surface due to moderate electrostatic interaction, and excited electrons were transferred from QDs to dehydro-AA, resulting in fluorescence quenching up to $22 \%$. Figure $4 \mathrm{~B}$ shows the fluorescence intensity of cysteine-capped InP/ZnS QDs versus time. Figure 4 shows the nonlinear emission peak intensity of QDs with time.

Figure 5A shows the variation of PL spectra of cysteinecapped InP/ZnS QDs by the addition of DA at different concentrations of $5 \mathrm{nM}, 10 \mathrm{nM}, 20 \mathrm{nM}, 40 \mathrm{nM}, 80 \mathrm{nM}$, and $100 \mathrm{nM}$. The fluorescence intensity decreases with the increase of DA concentration up to $62 \%$ due to strong electrostatic interactions. More excited electrons were transfered from QDs to oxidized dopamine, resulting in higher fluorescence quenching compared to AA.

Figure 5B shows the corresponding response of normalized fluorescence intensity of cysteine-capped InP/ZnS QDs $\left(I_{0} / I\right)$ versus the concentration of DA $\left(I_{0}\right.$ and $I$ represent the $\mathrm{PL}$ intensity in the absence and presence of DA, respectively). Figure 5 shows the plot of fluorescence quenching of cysteine-capped InP/ZnS QDs versus the concentration of DA. The Stern-Volmer equation was applied to investigate the relationship between quenching efficiency of QDs and the concentration of DA: $\left(I_{0} / I\right)=1+\mathrm{Kq}[\mathrm{Q}]$, where $I_{0}$ and $I$ are the fluorescence intensities in the absence and presence of 

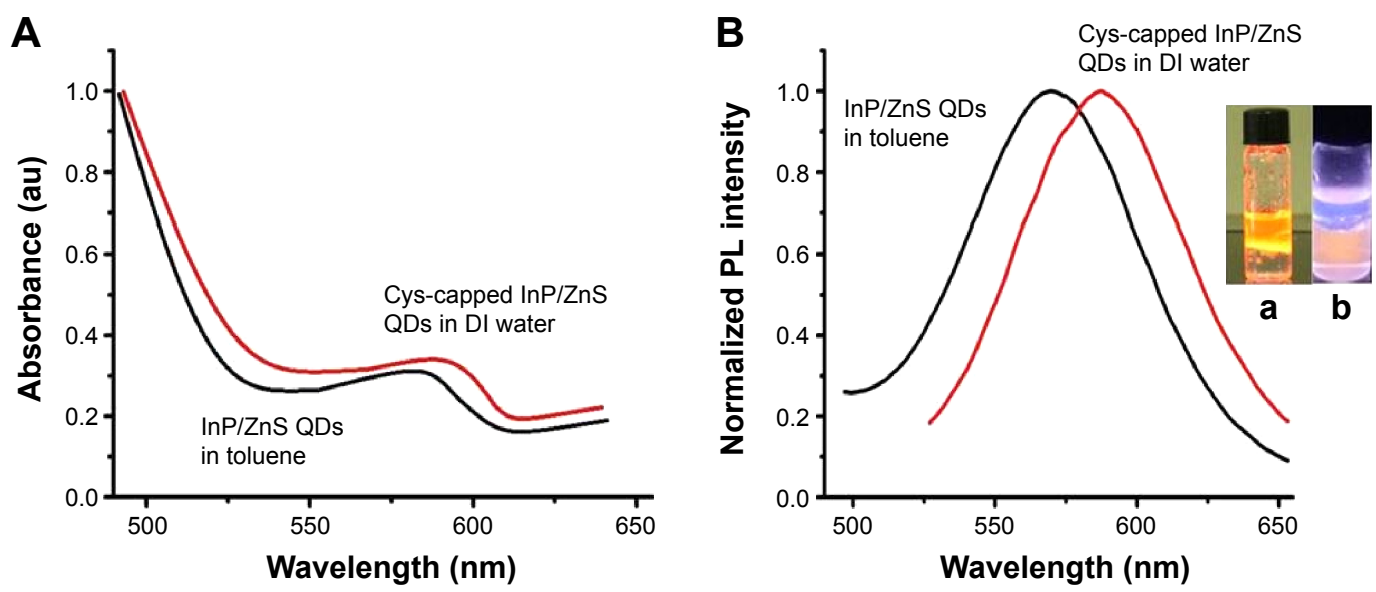

Figure 3 The optical absorption (A) and normalized PL spectra (B) of InP/ZnS QDs and L-cysteine-capped InP/ZnS QDs.

Note: Original InP/ZnS QDs were insoluble in water but these QDs were soluble in DI water after surface modification with L-Cys amino acid. That is why QDs were dispersed in two-layered mixture of toluene and DI water under UV illumination.

Abbreviations: QDs, quantum dots; UV, ultraviolet; Cys, cysteine; InP/ZnS QDs, indium phosphide/zinc sulfide quantum dots; PL, Photoluminescence; DI, deionized.
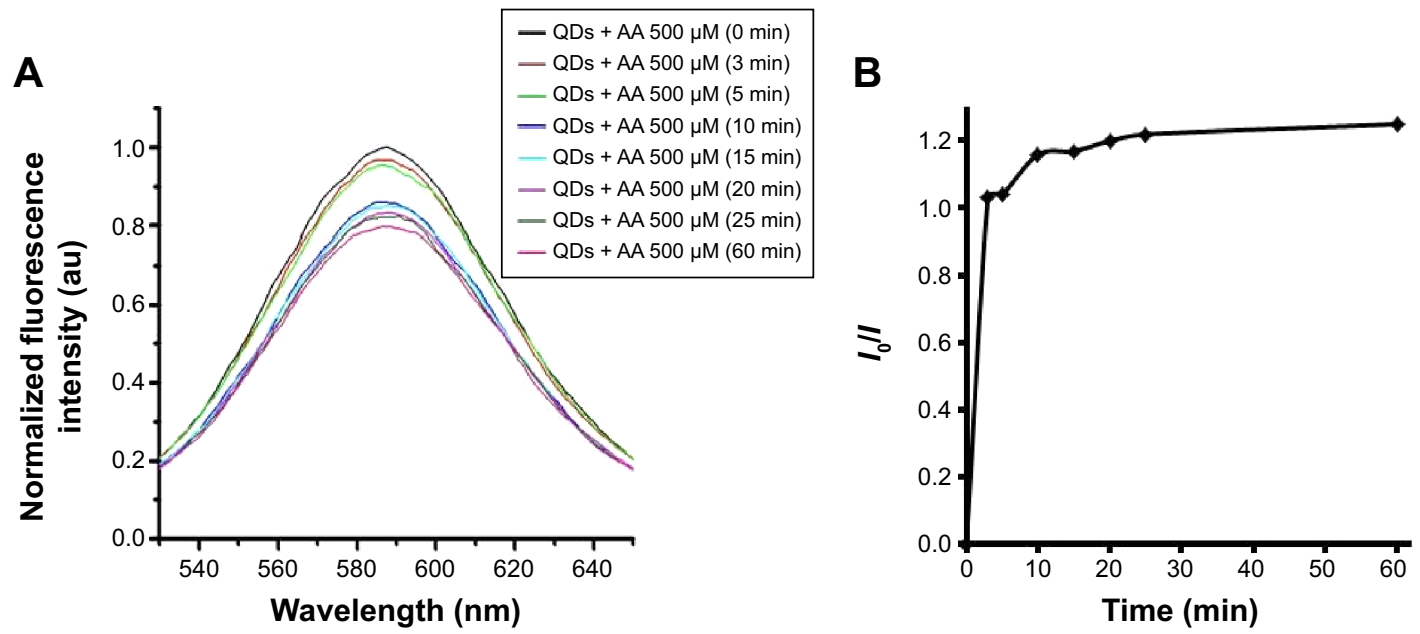

Figure 4 (A) PL spectra of cysteine-capped InP/ZnS QDs in the presence of AA and (B) the corresponding response of normalized fluorescence intensity versus time. Abbreviations: QDs, quantum dots; InP/ZnS QDs, indium phosphide/zinc sulfide quantum dots; AA, ascorbic acid; min, minutes; PL, photoluminescence.
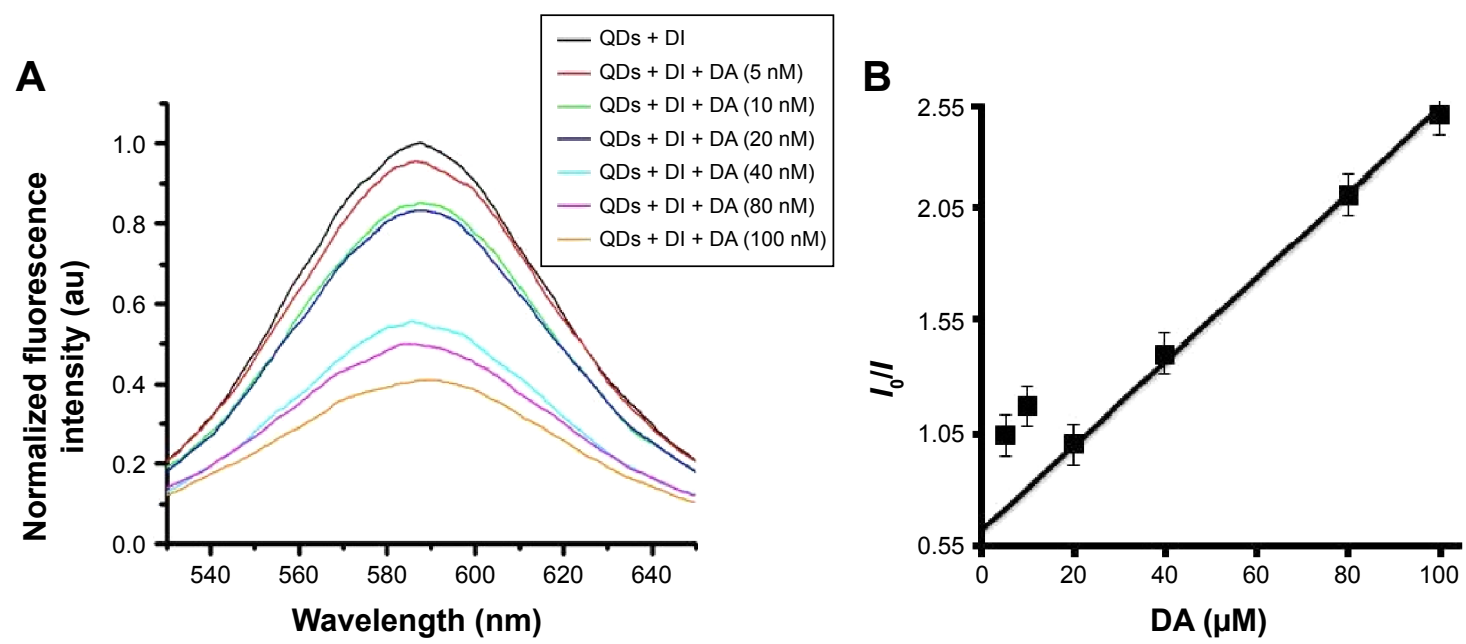

Figure 5 (A) PL spectra of cysteine-capped InP/ZnS QDs in the presence of DA and (B) the corresponding response of normalized fluorescence intensity versus concentration of DA. Notes: The figure displays (A) The PL spectra of cysteine-capped InP/ZnS QDs in the presence of DA and (B) the corresponding response of normalized fluorescence intensity cysteine-capped InP/ZnS QDs $\left(I_{0} / I\right)$ versus concentration of DA $\left(I_{0}\right.$ and $I$ represent the PL intensity in the absence and presence of DA, respectively). The figure displays the plot of relative fluorescence intensity of cysteine-capped InP/ZnS QDs $\left(I_{0} / I\right)$ against the concentration of DA, showing a linear fit to the Stern-Volmer equation. Abbreviations: QDs, quantum dots; InP/ZnS QDs, indium phosphide/zinc sulfide quantum dots; DA, dopamine (3,4-dihydroxyphenethylamine); PL, photoluminescence; DI, deionized. 


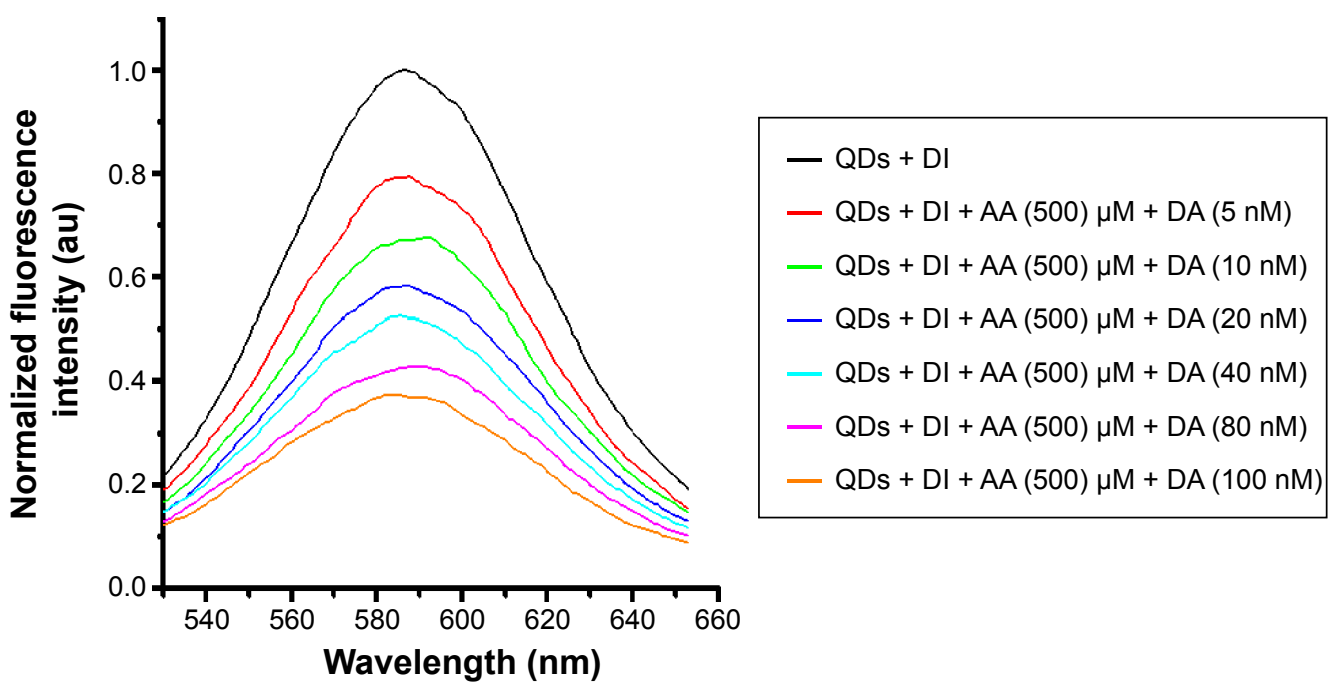

Figure $6 \mathrm{PL}$ spectra of cysteine-capped InP/ZnS QDs with various concentrations of DA in the presence of AA.

Abbreviations: QDs, quantum dots; InP/ZnS QDs, indium phosphide/zinc sulfide quantum dots; DA, dopamine (3,4-dihydroxyphenethylamine); PL, photoluminescence; $\mathrm{AA}$, ascorbic acid; DI, deionized.

analyte, respectively. [Q] is the concentration of the analyte, and $\mathrm{Kq}$ is the quenching constant of the analyte. As shown in Figure 5B, the linear relationship between $I_{0} / I$ and the concentration of DA could be observed when the concentration of DA was $<100 \mathrm{nM}$ with a standard deviation $R=0.984$. The limit of detection for DA was calculated to be as low as $875 \mathrm{pM}$. These results indicated that the evolution of fluorescence intensity is suitable for the determination of DA within a wide range of $800 \mathrm{pM}$ to $100 \mathrm{nM}$. Figure 6 shows the fluorescence quenching of cysteine-capped InP/ZnS QDs by DA at various concentrations $(5 \mathrm{nM}, 10 \mathrm{nM}, 20 \mathrm{nM}, 40 \mathrm{nM}$, $80 \mathrm{nM}$, and $100 \mathrm{nM})$ in the presence of AA $(500 \mu \mathrm{M})$. In this experiment, the fluorescence of QDs was quenched by AA at first, and after stabilization of fluorescence intensity, quenching by DA was investigated by the addition of DA. Up to $65 \%$ of quenching was observed.

Figure 7 shows the schematic illustration of fluorescence quenching of QDs by AA and DA. The hydroxyl group of

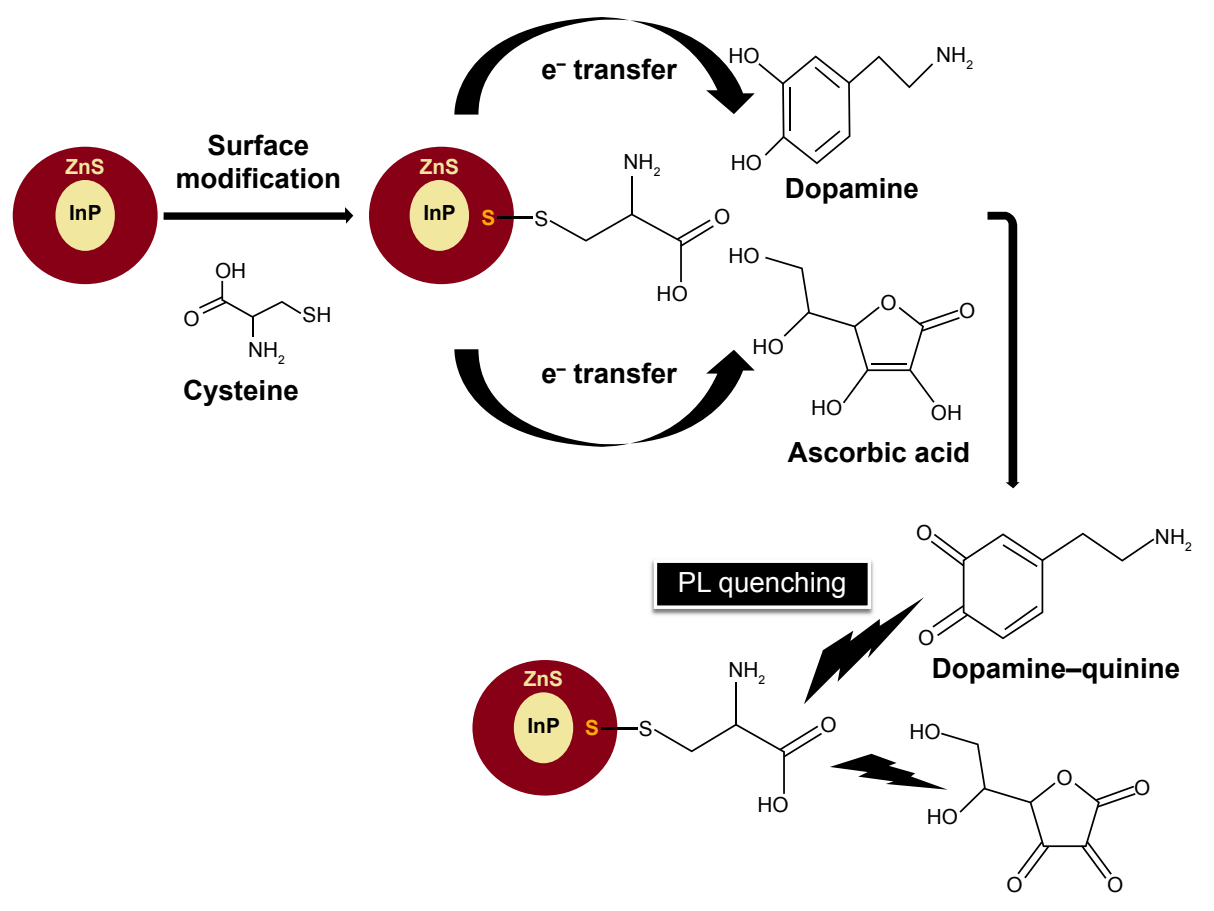

Dehydroascorbic acid

Figure 7 Schematic illustration of the fluorescence quenching of cysteine-capped $\ln P / Z n S Q D s$ in the presence of $A A$ and DA.

Abbreviations: InP/ZnS QDs, indium phosphide/zinc sulfide quantum dots; PL, photoluminescence; AA, ascorbic acid; DA, dopamine (3,4-dihydroxyphenethylamine). 


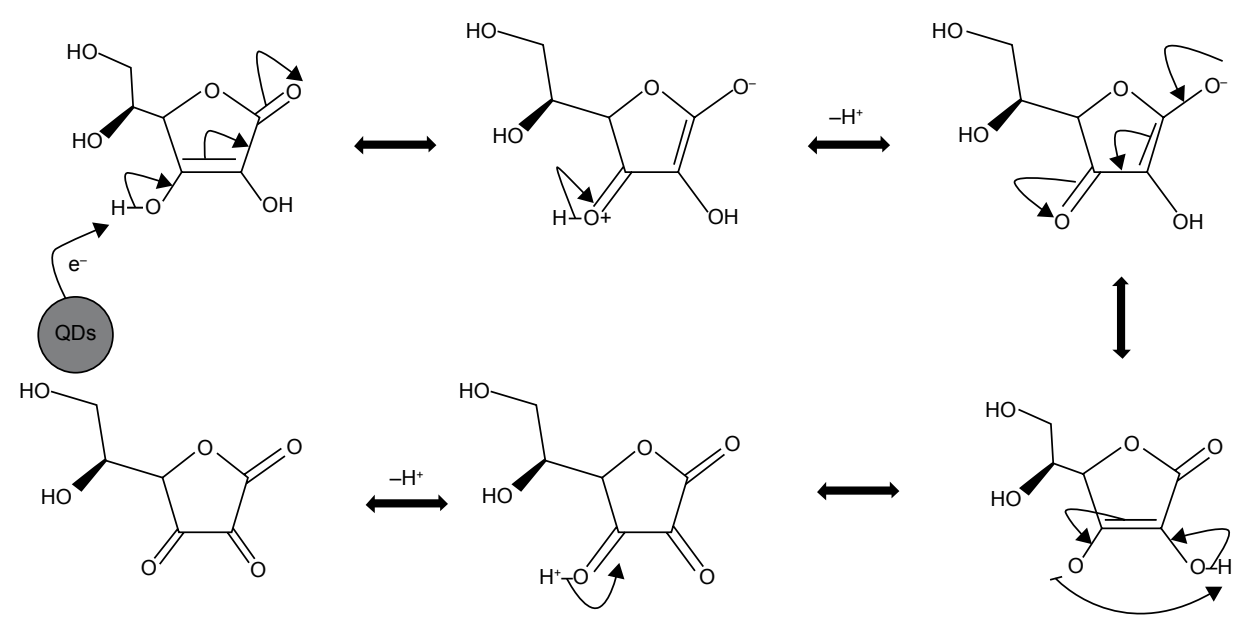

Ascorbic acid-enolate resonance structures

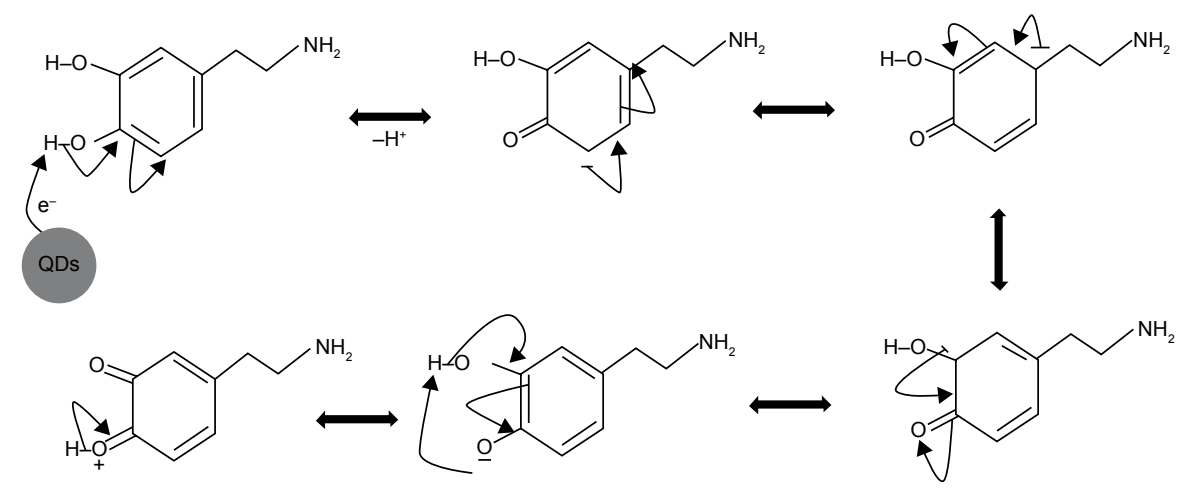

$-\mathrm{H}^{+} \downarrow$<smiles>NCCC1=CC(=O)C(=O)C=C1</smiles>

Dopamine-enolate resonance structures

Figure 8 Schematic representation of ascorbic acid and dopamine-enolate ion resonance structures.

Abbreviation: QDs, quantum dots.

catechol ring of DA and ribose ring of AA can be oxidized and acts as a hole trap to quench the fluorescence of QDs. When electrons are transferred from CB of QDs to DA and AA, enolate intermediate ions are formed. Figure 8 shows the schematic representation of AA and dopamine-enolate ion resonance structures. Dopamine and AA is slightly acidic for it to have recognizable acidic properties due to active hydroxyl group on the benzene and ribose rings. When electrons are transferred from QDs to DA and AA, enolate intermediate ions are formed. Dopamine and AA can lose a hydrogen ion because the phenoxide and ascorbate ions were stabilized to some extent. The negative charge on the oxygen atom is delocalized around the ring. The more stable the ion is, the more likely it is to form. One of the lone pairs on the oxygen atom overlaps with the delocalized electrons on the benzene and ribose rings. This overlap leads to a delocalization, which extends from the ring out over the oxygen atom. The dopamine enolate ion is more stable than the ascorbate enolate ion due to its more resonant structures. Due to this stability of enolate intermediate ions, the fluorescence of QDs was quenched more in the presence of DA than AA.

\section{Conclusion}

Water-soluble InP/ZnS QDs were prepared by the surface modification of L-Cys. FTIR spectra indicate that the S-H bond was cleaved and a new $\mathrm{S}-\mathrm{S}$ bond was formed between 
the L-Cys and InP/ZnS QDs. The fluorescence of the QDs is quenched by both AA and dopamine. The fluorescence quenching of QDs by dopamine was higher. The fluorescence of cysteine-capped InP/ZnS QDs was quenched by dopamine at various concentrations in the presence of AA. The analytical performance of this assay for DA was assessed, and it was found that the limit of detection was $875 \mathrm{pM}$.

\section{Acknowledgment}

This work was supported by the National Research Foundation (NRF) grant funded by the Korean government (MEST) (NRF-2006-2005382).

\section{Disclosure}

The authors report no conflicts of interest in this work.

\section{References}

1. Wood V, Halpert JE, Panzer MJ, Bawendi MG, Bulović V. Alternating current driven electroluminescence from $\mathrm{ZnSe} / \mathrm{ZnS}: \mathrm{Mn} / \mathrm{ZnS}$ nanocrystals. Nano Lett. 2009;9:2367-2371.

2. Klimov VI, Mikhailovsky AA, Xu S, et al. Optical gain and stimulated emission in nanocrystal quantum dots. Science. 2000;290:314-317.

3. Dong B, Cao L, Su G, Liu W, Qu H, Jiang D. Synthesis and characterization of the water-soluble silica-coated $\mathrm{ZnS}$ :Mn nanoparticles as fluorescent sensor for $\mathrm{Cu}^{2+}$ ions. J Colloid Interface Sci. 2009;339:78-82.

4. Xu B, Cai B, Liu M, Fan H. Microwave synthesis of water-dispersed cdte/cds/zns core-shell-shell quantum dots with excellent photostabilityand biocompatibility. Adv Mater. 2008;20:3416-3421.

5. Durgadas CV, Sreenivasan K, Sharma CP. Bright blue emitting CuSe/ $\mathrm{ZnS} /$ silica core/shell/shell quantum dots and their biocompatibility. Biomaterials. 2012;33:6420-6429.

6. Lin L, Chen J, Yao H, Chen Y, Zheng Y, Lin X. Simultaneous determination of dopamine, ascorbic acid and uric acid at poly (Evans Blue) modified glassy carbon electrode. Bioelectrochemistry. 2008;73:11-17.

7. Kalimuthu P, John SA. Electropolymerized film of functionalized thiadiazole on glassy carbon electrode for the simultaneous determination of ascorbic acid, dopamine and uric acid. Bioelectrochemistry. 2009;77: $13-18$.

8. Giros B, Caron MG. Molecular characterization of the dopamine transporter. Trends Pharmacol Sci. 1993;14:43-43.

9. Usiello A, Baik JH, Rougé-Pont F, et al. Distinct functions of the two isoforms of dopamine D2 receptors. Nature. 2000;408:199-203.

10. Darvas M, Palmiter RD. Restricting dopaminergic signaling to either dorsolateral or medial striatum facilitates cognition. J Neurosci. 2010; 30:1158-1165.
11. Robinson DL, Hermans A, Seipel AT, Wightman RM. Monitoring rapid chemical communication in the brain. Chem Rev. 2008;108: 2554-2584.

12. Nikolaus S, Antke C, Müller H-W. In vivo imaging of synaptic function in the central nervous system: I. Movement disorders and dementia. Behav Brain Res. 2009;204:1-31.

13. Yi S-Y, Chang H-Y, Cho H-H, Park YC, Lee SH, Bae ZU. Resolution of dopamine and ascorbic acid using nickel(II) complex polymer-modified electrodes. J Electroanal Chem. 2007;602:217-225.

14. George O, Moal ML, Koob GF. Allostasis and addiction: role of the dopamine and corticotropin-releasing factor systems. Physiol Behav. 2012;106:58-64

15. O'Neill RD. Microvoltammetric techniques and sensors for monitoring neurochemical dynamics in vivo. A review. Analyst. 1994;119: 767-779.

16. Capella P, Ghasemzadeh MB, Mitchell K, Adams RN. Nafion-coated carbon fiber electrodes for neurochemical studies in brain tissue. Electroanalysis. 1990;2:175-182.

17. Li L, Liu H, Shen Y, Zhang J, Zhu JJ. Electrogenerated chemiluminescence of Au nanoclusters for the detection of dopamine. Anal Chem. 2011;83:661-665.

18. Liu Q, Zhu X, Huo Z, He X, Liang Y, Xu M. Electrochemical detection of dopamine in the presence of ascorbic acid using PVP/graphene modified electrodes. Talanta. 2012;97:557-562.

19. Zhao Y, Zhao S, Huang J, Ye F. Quantum dot-enhanced chemiluminescence detection for simultaneous determination of dopamine and epinephrine by capillary electrophoresis. Talanta. 2012;85:2650-2654.

20. Liu L, Li S, Liu L, Deng D, Xia N. Simple, sensitive and selective detection of dopamine using dithiobis (succinimidyl propionate)modified gold nanoparticles as colorimetric probes. Analyst. 2012; 137:3794-3799.

21. Nikolajsen RPH, Hansen ÅM. Analytical methods for determining urinary catecholamines in healthy subjects. Anal Chim Acta. 2001;449: $1-15$.

22. Zhang L, Teshima N, Hasebe T, Kurihara M, Kawashima T. Flowinjection determination of trace amounts of dopamine by chemiluminescence detection. Talanta. 1999;50:677-683.

23. Zhou Z, Wang Q. An efficient optical-electrochemical dual probe for highly sensitive recognition of dopamine based on terbium complex functionalized reduced graphene oxide. Nanoscale. 2014;6: 4583-4586.

24. Li Z, Li W, Cheng Y, Hao L. Chemiluminescent detection of DNA hybridization and single-nucleotide polymorphisms on a solid surface using target-primed rolling circle amplification. Analyst. 2008;133: 1164-1168.

25. Zhao D, Song H, Hao L, Liu X, Zhang L, Lv Y. Luminescent ZnO quantum dots for sensitive and selective detection of dopamine. Talanta. 2013;107:133-139.

26. Ariffin N, Yusof NA, Abdullah J, et al. Synthesis and surface modification of biocompatible water soluble core-shell quantum dots. Adv Mater Res. 2014;879:184-190.
International Journal of Nanomedicine

\section{Publish your work in this journal}

The International Journal of Nanomedicine is an international, peerreviewed journal focusing on the application of nanotechnology in diagnostics, therapeutics, and drug delivery systems throughout the biomedical field. This journal is indexed on PubMed Central, MedLine, CAS, SciSearch ${ }^{\circledR}$, Current Contents ${ }^{\circledR} /$ Clinical Medicine,

\section{Dovepress}

Journal Citation Reports/Science Edition, EMBase, Scopus and the Elsevier Bibliographic databases. The manuscript management system is completely online and includes a very quick and fair peer-review system, which is all easy to use. Visit http://www.dovepress.com/ testimonials.php to read real quotes from published authors. 